\title{
Approximate Method for Solving the Linear Fuzzy Delay Differential Equations
}

\author{
S. Narayanamoorthy and T. L. Yookesh \\ Department of Applied Mathematics, Bharathiar University, Coimbatore 641 046, India \\ Correspondence should be addressed to S. Narayanamoorthy; snm_phd@yahoo.co.in
}

Received 7 September 2015; Accepted 8 October 2015

Academic Editor: Carlo Bianca

Copyright ( 2015 S. Narayanamoorthy and T. L. Yookesh. This is an open access article distributed under the Creative Commons Attribution License, which permits unrestricted use, distribution, and reproduction in any medium, provided the original work is properly cited.

\begin{abstract}
We propose an algorithm of the approximate method to solve linear fuzzy delay differential equations using Adomian decomposition method. The detailed algorithm of the approach is provided. The approximate solution is compared with the exact solution to confirm the validity and efficiency of the method to handle linear fuzzy delay differential equation. To show this proper features of this proposed method, numerical example is illustrated.
\end{abstract}

\section{Introduction}

Fuzzy set theory is a powerful tool for modeling uncertainty and for processing vague or subjective information in mathematical models, which has been applied to a wide variety of real problems. Thus the theory of fuzzy differential equation has attracted widespread attention and has been rapidly growing. It is massively studied by many researchers $[1,2]$. The theory of fuzzy differential equations has undergone a rapid evolution in the last three decades because the idea of fuzzy set theory is simple and natural. The concept of fuzzy was first introduced by Zadeh [3]. Chang and Zadeh [4] had introduced many concepts in fuzzy derivatives. Fuzzy delay differential equations have a wide range of applications in real time applications of control theory, physics, ecology, economics, population study, inventory control, and the theory of nuclear reactors. It is difficult to obtain exact solutions of fuzzy differential equations and hence several numerical methods were proposed [5-8]. Abbasbandy and Allahviranloo [9] developed numerical algorithms for solving fuzzy differential equations based on Seikkala's derivative [10]. Jafari et al. [11] worked on the solving $n$th order fuzzy differential equations by the variational iteration method. Allahviranloo et al. [12] used the predictor-corrector method to find the numerical solution of fuzzy differential equations.
Delay differential equations are the type of differential equations in which the derivative of the unknown function at a certain time is given in terms of the values of the function at a previous time.

Delay differential equations and ordinary differential equations are used to describe physical phenomena, but they are different. While in ordinary differential equations the derivatives of unknown functions are dependent only on the current value of the independent variable, in delay differential equations the derivatives of unknown functions are dependent on the values of the functions at previous time. This implies that the solution of delay differential equations requires the knowledge of the current state and also the certain previous time state. For example, the present state of change of unknown functions depends upon the past values of the same functions in both physical and biological systems.

Linear differential equations with retarded argument concept proposed by Myškis (1951) [13] and later many researchers continued to work on these concepts [14, 15]. Driver [15] wrote a book about ordinary differential equations and delay differential equations which explains the equations clearly. Bellen and Zennaro [16] presented the numerical solutions to delay differential equations. Khastan et al. [17] discussed the concepts of fuzzy delay differential equations 
under the generalized differentiability. Barzinji et al. [18] studied the linear fuzzy delay differential systems to analyze the stability of steady state. For solving the delay differential equations Ibrahim et al. [19] used the $2-h$ step spline method. Evans and Raslan [20] solved delay differential equation by the domain decomposition method. Shakeri and Dehghan [21] found the solution to delay differential equation by the homotopy perturbation method. Mirzaee and Latifi [22] used the differential transform method to the delay differential equation. For the delay differential equation Sadehgi Hafshejani et al. [23] discussed the Legendre Wavelet method. Numerical and theoretical treatment for both linear and nonlinear delay differential equations using the variational iteration method was proposed by Khader [24]. Guerrini worked on delay differential AK model with nonpositive population growth rate [25] and the time delay effects on the qualitative behavior of an economic growth model [26]. Akgül and Kiliçman [27] solved delay differential equations by an accurate method with interpolation. Abdul Aziz et al. [28] derived delay differential equations of small and vanishing lag using the multistep block method. The general form of the delay differential equation is given as follows:

$$
\begin{aligned}
y^{\prime}(t) & =f\left(t, y(t), y\left(t-r_{1}\right), \ldots, y_{n}\left(t-r_{i}\right)\right), \quad t \geq t_{0} \\
y(t) & =\phi(t), \quad t \geq t_{0}
\end{aligned}
$$

where $r_{i}$ is constant for each $i=1,2,3, \ldots, n$.

Let us consider the fuzzy delay differential equation as follows:

$$
\begin{array}{ll}
\underline{y^{\prime}}(t)=f\left(t, \underline{y}(t), \underline{y}\left(t-r_{1}\right), \ldots, \underline{y_{n}}\left(t-r_{i}\right)\right), & \\
& t \geq t_{0}, \\
\underline{y}(t)=\phi(t), \quad t \geq t_{0}, & \\
\overline{y^{\prime}}(t)=f\left(t, \bar{y}(t), \bar{y}\left(t-r_{1}\right), \ldots, \overline{y_{n}}\left(t-r_{i}\right)\right), \quad t \geq t_{0}, \\
\bar{y}(t)=\phi(t), \quad t \geq t_{0},
\end{array}
$$

where $r_{i}$ is constant for each $i=1,2,3, \ldots, n$.

The Adomian decomposition method is named after Adomian [29]. The Adomian decomposition method is well suited to solve Cauchy problems. The advantage of the method is that it can be applied directly for all types of differential and integral equations, linear or nonlinear, homogeneous or inhomogeneous, and with constant coefficients or with variable coefficients. Another important advantage is that the method is capable of greatly reducing the size of computational work while still maintaining high accuracy of the numerical solution. This method is based on the decomposition of a solution of a nonlinear operator equation in a series of functions. Each of the series is obtained from an expansion of an analytic function into an abstract formulation, but the difficulty arises in calculating the polynomial and in proving the convergence in the series of functions. The Adomian decomposition method is used to solve a wide range of physical problems in various engineering fields such as wave and heat and mass transfer equations. Thus, many researchers have applied and solved their problems by using Adomian decomposition method.

Motivated by the work of Evans and Raslan [20], in this paper, the author proposes an approximate method to solve the linear fuzzy delay differential equations using Adomian decomposition method. The purpose of this paper is to find how this technique works on delay differential equations under fuzzy environment. The Adomian decomposition method converges rapidly to form the exact solution. This method does not involve any discretization of variables and hence it is free from rounding off errors. We illustrate a numerical example to explain the proposed method.

\section{Preliminaries}

In this section, we present the most basic notations and definitions, which are used throughout this work. We start with defining a membership function.

Definition 1 (see [30]). Let $X$ be a nonempty set. A fuzzy set $\bar{A} \in X$ is characterized by its membership function $\mu_{\bar{A}}: X \rightarrow$ $[0,1]$ and $\mu_{\bar{A}(x)}$ is interpreted as the degree of membership of element $x$ in fuzzy set $\bar{A}$ for each $x \in X$. It is clear that $\bar{A}$ is determined by the set of tuples $\bar{A}=\left(x, \mu_{\bar{A}(x)}\right) \mid x \in X$.

Definition 2. A fuzzy number is a map $u: R \rightarrow I=[0,1]$ which satisfies the following three conditions:

(i) $u$ is upper semicontinuous.

(ii) $u(x)=0$ outside some interval $[c, d] \subset R$.

(iii) There exist real numbers $a, b$ such that $c \leq a \leq b \leq d$, where

(1) $u(x)$ is monotonic increasing on $[c, d]$,

(2) $u(x)$ is monotonic decreasing on $[b, d]$,

(3) $u(x)=1, a \leq x \leq b$.

The set of all such fuzzy numbers is represented by $E^{\prime}$.

Definition 3. Given a fuzzy set $\bar{A}$ defined on $X$ and a number $\alpha \in[0,1]$, the $\alpha$-cut, $\bar{A}^{\alpha}$, and the strong $\alpha$-cut, $\bar{A}^{\alpha+}$, are the crisp sets

$$
\begin{aligned}
\bar{A}^{\alpha} & =\{x \mid A(x) \geq \alpha\}, \\
\bar{A}^{\alpha+} & =\{x \mid A(x)>\alpha\} .
\end{aligned}
$$

Unlike in the conventional set theory, convexity of fuzzy sets refers to properties of the membership function rather than to the support of a fuzzy set.

Definition 4. An arbitrary fuzzy number is parametric form represented by an ordered pair of functions $(\underline{u}(\alpha), \bar{u}(\alpha)), 0 \leq$ $\alpha \leq 1$, which satisfy the following requirements:

(1) $\underline{u}(\alpha)$ is a bounded left continuous nondecreasing function over $[0,1]$, 
(2) $\bar{u}(\alpha)$ is a bounded left continuous nonincreasing function over $[0,1]$,

(3) $\underline{u}(\alpha) \leq \bar{u}(\alpha), 0 \leq \alpha \leq 1$.

For arbitrary $u(\alpha)=(\underline{u}(\alpha), \bar{u}(\alpha)), v(\alpha)=(\underline{v}(\alpha), \bar{v}(\alpha))$ and scalar $k$, we define addition $((\bar{u})+(\bar{v}))$, subtracting by $k$ as

(1) Addition:

$$
\begin{aligned}
& \underline{(u+v)}(\alpha)=\underline{u}(\alpha)+\underline{v}(\alpha), \\
& \overline{(u+v)}(\alpha)=\bar{u}(\alpha)+\bar{v}(\alpha),
\end{aligned}
$$

(2) Subtraction:

$$
\begin{aligned}
& \underline{(u-v)}(\alpha)=\underline{u}(\alpha)-\underline{v}(\alpha), \\
& \overline{(u-v)}(\alpha)=\bar{u}(\alpha)-\bar{v}(\alpha),
\end{aligned}
$$

(3) Scalar multiplication:

$$
k(x)= \begin{cases}(k \underline{u}(\alpha), k \bar{u}(\alpha)), & k \geq 0, \\ (k \bar{u}(\alpha), k \underline{u}(\alpha)), & k<0 .\end{cases}
$$

Definition 5. A fuzzy function $f(x)$ is a fuzzy set on $y$ with membership function

$$
\mu_{f(x)}(y)=\mu_{f}(x, y) .
$$

Its inverse $f^{-1}(y)$ is a fuzzy set on $x$ with

$$
\mu_{f^{-1}}(y)=\mu_{f}(x, y) \text {. }
$$

Definition 6. Suppose that $f:[c, d] \rightarrow F$. For each partition $p=x_{0}, x_{1}, \ldots, x_{n}$ of $[c, d]$ and for arbitrary points $\xi_{i}, x_{i-1} \leq$ $\xi_{i} \leq x_{i}$, let

$$
R_{p}=\sum_{i=1}^{n} f\left(\xi_{i}\right) \Delta_{i}
$$

where $\Delta=x_{i}-x_{i-1}$. Then the definite integral $\int_{c}^{d} f(r) d r$ of $f$ over $[c, d]$ is defined by

$$
\int_{c}^{d} f(x) d x=\lim _{|p| \rightarrow 0} R_{p}
$$

provide the limits exist.

Definition 7. A fuzzy set $\bar{A}$ is the triangular fuzzy number with peak (or center) $a$, left width $\alpha>0$ and right $\beta>0$ (Figure 1) if its membership function has the following form:

$$
\mu(x)= \begin{cases}1-\frac{a-x}{\alpha} & \text { if } a-\alpha<x<a, \\ 1-\frac{x-a}{\beta} & \text { if } a<x<a+\beta, \\ 0 & \text { otherwise. }\end{cases}
$$

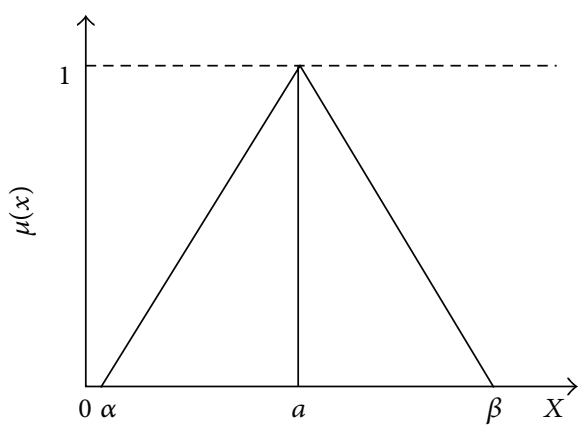

FIGURE 1: Triangular fuzzy number.

Definition 8. Let $G$ be an analytical function $\sum u_{n}$ a convergent series in $E$. The Adomian polynomials are defined by

$$
A_{k}=\frac{1}{k !}\left(\frac{d^{k}}{d \lambda^{k}} G\left(\sum_{n=0}^{\infty} u_{n} \lambda^{n}\right)\right)_{\lambda=0} .
$$

Definition 9. Let $x, y \in R_{F}$. If there exists $z \in R_{f}$ such that $x=y+z$, then $z$ is called the Hukuhara difference of $x, y$ and it is denoted by $x \ominus y$.

\section{Fuzzy Delay Differential Equation}

In this section, we refine fuzzy initial value problem [9] for delay differential equation using Sekkiala derivatives [10] as follows:

$$
\begin{aligned}
& y^{\prime}(x)=f(x, y(x), y(x-t)), \quad t \in\left[x_{0}, X\right], \\
& y\left(x_{0}\right)=y_{0},
\end{aligned}
$$

where $y$ is a fuzzy function of $x, f(x, y, y(x-t))$ is a fuzzy function of the crisp variable $x$, and $y\left(x_{0}\right)=y_{0}$ is a triangular shaped fuzzy number; therefore we have a fuzzy Cauchy problem.

We denote the fuzzy function $y=[y, \bar{y}]$. It means the $\alpha$-level set of $y(x)$ for $x \in\left[x_{0}, X\right]$ is

$$
\begin{aligned}
{\left[y\left(x_{0}\right)\right]_{\alpha} } & =\left[\underline{y}\left(x_{0} ; \alpha\right), \bar{y}\left(x_{0} ; \alpha\right)\right], \\
{[y(x)]_{\alpha} } & =[\underline{y}(x ; \alpha), \bar{y}(x ; \alpha)], \\
{[y(x-t)]_{\alpha} } & =[\underline{y}(x-t ; \alpha), \bar{y}(x-t ; \alpha)],
\end{aligned}
$$

$\alpha \in[0,1]$.

By using the extension principle of Zadeh, we have the membership function

$$
\begin{aligned}
& f(x, y(x))(s)=\sup \left\{\frac{y(x)(\tau)}{s}=f(x, \tau) x \in R\right\}, \\
& f(x, y(x-t))(s) \\
& \quad=\sup \left\{\frac{y(x-t)(\tau)}{s}=f(x-t, \tau) x-t \in R\right\}
\end{aligned}
$$


so $f(x, y(x), y(x-t))$ is a fuzzy number. From this it follows that

$$
\begin{aligned}
& {[f(x, y(x), y(x-t))]_{\alpha}} \\
& \quad=f_{1}[(x, y(x), y(x-t) ; \alpha)] \\
& \quad \cdot f_{2}[(x, y(x), y(x-t) ; \alpha)], \quad \alpha \in[0,1],
\end{aligned}
$$

where

$$
\begin{aligned}
& {\left[f_{1}(x, y(x), y(x-t)) ; \alpha\right]=\min \left\{\frac{f(x, u)}{u}\right.} \\
& \in[\underline{y}(x ; \alpha), \bar{y}(x ; \alpha), \underline{y}(x-t ; \alpha), \bar{y}(x-t ; \alpha)]\}, \\
& {\left[f_{2}(x, y(x), y(x-t)) ; \alpha\right]=\max \left\{\frac{f(x, u)}{u}\right.} \\
& \quad \in[\underline{y}(x ; \alpha), \bar{y}(x ; \alpha), \underline{y}(x-t ; \alpha), \bar{y}(x-t ; \alpha)]\} ;
\end{aligned}
$$

we define

$$
\begin{aligned}
& \quad[F(x, y(x), y(x-t)) ; \alpha] \\
& \quad=F[x, \underline{y}(x ; \alpha), \bar{y}(x ; \alpha), \underline{y}(x-t ; \alpha), \bar{y}(x-t ; \alpha)], \\
& {[G(x, y(x), y(x-t)) ; \alpha]} \\
& \quad=G[x, \underline{y}(x ; \alpha), \bar{y}(x ; \alpha), \underline{y}(x-t ; \alpha), \bar{y}(x-t ; \alpha)] .
\end{aligned}
$$

Theorem 10 (see [10]). Let $f$ satisfy

$$
|f(x, v)-f(x, \bar{v})| \leq g(x,|v-\bar{v}|), \quad x \geq 0, v, \bar{v} \in R,
$$

where $g: R_{+} \times R_{+} \rightarrow R_{+}$is a continuous mapping such that $\alpha \rightarrow g(x, \alpha)$ is nondecreasing and the initial value problem

$$
\begin{aligned}
& u^{\prime}(t)=g(x, u(x)), \\
& u(0)=0,
\end{aligned}
$$

has the solution on $R_{+}$for $u_{0}>0$ and that $u(t)=0$ is the only solution of (20) for $u_{0}=0$. Then the fuzzy initial value problem (13) has a unique solution.

\section{Analysis of the Method}

Consider the differential equation written in the following form:

$$
L y+N y=g(x),
$$

where $L$ is linear operator, $N$ is a nonlinear operator, and $g(x)$ is a nonhomogeneous term. If differential equations are described by $M$ order, where the differential operation $L$ is given by [31]

$$
L(\cdot)=\frac{d^{M}(\cdot)}{d x^{M}},
$$

and the inverse operator $L^{-1}$ is therefore considered as $M$-fold integral operator defined by

$$
L^{-1}(*)=\underbrace{\int_{0}^{x} \int_{0}^{x} \cdots \int_{0}^{x}(*) d x \cdots d x}_{M},
$$

then

$$
y=L^{-1}(*)(g(x)-N y) .
$$

The Adomian's method consists of approximating the solution of (24) as an infinite series

$$
y=\sum_{n=0}^{\infty} y_{n}(x)
$$

and decomposing the nonlinear operator $N$ as

$$
N y=\sum_{n=0}^{\infty} A_{n}
$$

where $A_{n}$ are Adomian polynomials of $y_{0}, y_{1}, \ldots, y_{n}$ given by

$$
A_{n}=\frac{1}{n} \frac{d^{n}}{d \mu^{n}}\left[N\left(\sum_{i=0}^{\infty} \mu^{i} y_{i}\right)\right]_{\mu=0}
$$

$n=0,1,2, \ldots$ substituting the derivatives (25), (26), and (27) in (24) it yields

$$
\sum_{i=0}^{\infty} y_{n}(x)=L^{-1}(g(x))-L^{-1}\left(\sum_{n=0}^{\infty} A_{n}\right) .
$$

Thus, we can identify

$$
\begin{aligned}
y_{0} & =L^{-1}(g(x)), \\
y_{n+1} & =L^{-1}\left(\sum_{n=0}^{\infty} A_{n}\right)=A_{n}\left(y_{0}, y_{1}, \ldots, y_{n}\right),
\end{aligned}
$$

where $n=0,1,2, \ldots$ We then define the $K$-term approximate to the solution $y$ by

$$
\begin{aligned}
\Phi_{K}[y] & =\sum_{n=0}^{K} y_{n}, \\
\lim _{K \rightarrow \infty} \Phi_{K}[y] & =y .
\end{aligned}
$$

Practical formula for the calculation of Adomian decomposition polynomials is given in $A_{n}$. However all terms of the series cannot be determined usually by using $A_{n}=\sum_{i=0}^{\infty}$ approximated with truncated series $\Phi_{k}=y_{0}+y_{1}+\cdots+y_{n-1}$ as the above result (30).

\section{The Algorithm of Approximate Solution and Its Applications}

Analysis of the method is discussed in Section 4. The algorithm of fuzzy delay differential equation under Adomian decomposition method is presented in Section 5.1. 
5.1. Approximate Solutions of Fuzzy Delay Differential Equations by Applying the Adomian Decomposition Method. Consider the DDE $[20,31,32]$

$$
y(x)=f(x, y(x), y(x-t)), \quad 0 \leq x \leq 1
$$

with initial conditions

$$
\begin{aligned}
y^{(i)}(0) & =\left(\underline{y}_{0}^{i} ; \bar{y}_{0}^{i}\right), \quad i=0,1,2, \ldots, N-1 \\
y(x) & =\phi(x), \quad x \leq 0 .
\end{aligned}
$$

Let $Y=\left[Y_{1}, Y_{2}\right]$ be the exact solution and let $y=\left[y_{1}, y_{2}\right]$ be the approximate solution of the given equation

$$
\begin{aligned}
& {[Y(x)]_{\alpha}=\left[Y_{1}(x, x-t ; \alpha), Y_{2}(x, x-t ; \alpha)\right],} \\
& {[y(x)]_{\alpha}=\left[y_{1}(x, x-t ; \alpha), y_{2}(x, x-t ; \alpha)\right],}
\end{aligned}
$$

keeping argument the value of $\alpha$ fixed; then the exact and approximate solution of $x_{n}$ are represented by

$$
\begin{aligned}
{[Y(x)]_{\alpha} } & =\left[Y_{1}(x, x-t ; \alpha), Y_{2}(x, x-t ; \alpha)\right], \\
{[y(x)]_{\alpha} } & =\left[y_{1}(x, x-t ; \alpha), y_{2}(x, x-t ; \alpha)\right], \\
& 0 \leq n \leq N .
\end{aligned}
$$

Now, the approximate solution is solved by the proposed Adomian decomposition method. We arrive at the following equation:

$$
\begin{array}{ll}
L \underline{y}(x ; \alpha)=f(x, \underline{y}(x), \underline{y}(x-t) ; \alpha), & 0 \leq x \leq 1, \\
L \bar{y}(x ; \alpha)=f(x, \bar{y}(x), \bar{y}(x-t) ; \alpha), & 0 \leq x \leq 1,
\end{array}
$$

where $L(\cdot)=d^{N}(\cdot) / d x^{N}$ is the differential operator. Operating on both sides of the above equation with the inverse operator (namely, $L^{-1}(*)=\iint \cdots \int(*) d x d x \cdots d x$ ) on using the given initial conditions, we have

$$
L^{-1}(\cdot)=\int_{x}^{0}(\cdot)_{N \text { times }} d x
$$

therefore in operating with $L^{-1}$ on (35) and we have

$$
\begin{aligned}
& \underline{y}(x ; \alpha)=\sum_{j=0}^{N-1} \frac{\alpha_{j}}{j !} x_{i}+L^{-1}(f(x, \underline{y}(x), \underline{y}(x-t))), \\
& \bar{y}(x ; \alpha)=\sum_{j=0}^{N-1} \frac{\alpha_{j}}{j !} x_{i}+L^{-1}(f(x, \bar{y}(x), \bar{y}(x-t))),
\end{aligned}
$$

where $\alpha_{i}$ is constant initial condition. In Adomain decomposition method, it is assumed that the unknown functions $y(x)$ can be expressed by an infinite series of the form

$$
\begin{aligned}
& \underline{y}(x)=\sum_{n=0}^{\infty} \underline{y}_{n} x, \\
& \bar{y}(x)=\sum_{n=0}^{\infty} \bar{y}_{n} x ;
\end{aligned}
$$

by these components, $\underline{y}_{n}(x)$ and $\bar{y}_{n}(x)$ are determined.
The nonlinear terms $f(x, \underline{y}(x), \underline{y}(x-t)) ; f(x, \bar{y}(x), \bar{y}(x-$ $t)$ ) are defined by the Adomian polynomials as

$$
\begin{aligned}
& f(x, \underline{y}(x), \underline{y}(x-t))=\sum_{n=0}^{\infty} A_{n}, \\
& f(x, \bar{y}(x), \bar{y}(x-t))=\sum_{n=0}^{\infty} B_{n},
\end{aligned}
$$

where $A_{n}$ and $B_{n}$ are Adomian polynomials that can be generated for all forms of non linearity [36] as

$$
\begin{aligned}
& A_{n} \\
& \quad=\frac{1}{n !} \frac{d^{n}}{d \mu^{n}}\left[f\left(x, \sum_{i=0}^{\infty} \mu^{i} \underline{y_{i}}(x), \sum_{i=0}^{\infty} \mu^{i} \underline{y_{i}}(x-t)\right)\right]_{\mu=0}, \\
& B_{n}=\frac{1}{n !} \frac{d^{n}}{d \delta^{n}}\left[f\left(x, \sum_{i=0}^{\infty} \delta^{i} \overline{y_{i}}(x), \sum_{i=0}^{\infty} \delta^{i} \overline{y_{i}}(x-t)\right)\right]_{\delta=0},
\end{aligned}
$$

on substituting (39) and (41) in (37) and (40) and (42) in (38), we have

$$
\begin{aligned}
& \sum_{n=0}^{\infty} \underline{y}_{n}(x ; \alpha)=\sum_{j=0}^{N-1} \frac{\alpha_{j}}{j !} x^{j}+L^{-1}\left(\sum_{n=0}^{\infty} A_{n}\right) ; \\
& \sum_{n=0}^{\infty} \bar{y}_{n}(x ; \alpha)=\sum_{j=0}^{N-1} \frac{\alpha_{j}}{j !} x^{j}+L^{-1}\left(\sum_{n=0}^{\infty} B_{n}\right)
\end{aligned}
$$

to determine the components $y_{n}(x ; \alpha) ; \bar{y}_{n}(x ; \alpha), n \geq 0$. We need to find the $\underline{y}_{0}(x ; \alpha)$ and $\bar{y}_{0}(x ; \alpha)$ zero component first by all terms that arise from the initial conditions at $x=0$ and from integrating the source term if it exists. Second, the remaining components of $y_{n}(x ; \alpha) ; \bar{y}_{n}(x ; \alpha)$ can be determined in a way such that each component is determined by using the preceding components. In other words, the method introduces the recursive relation

$$
\begin{aligned}
\underline{y}_{0}(x ; \alpha) & =\sum_{j=0}^{N-1} \frac{\alpha_{j}}{j !} x^{j}, \\
\underline{y}_{n+1}(x ; \alpha) & =L^{-1}\left(A_{n}\right), \quad n \geq 0 \\
\bar{y}_{0}(x ; \alpha) & =\sum_{j=0}^{N-1} \frac{\alpha_{j}}{j !} x^{j}, \\
\bar{y}_{n+1}(x ; \alpha) & =L^{-1}\left(B_{n}\right), \quad n \geq 0,
\end{aligned}
$$

for the determination of the components $\underline{y}_{n}(x ; \alpha) ; \bar{y}_{n}(x ; \alpha)$, $n \geq 0$ of $\bar{y}(x ; \alpha) ; \bar{y}(x ; \alpha)$, the series solution of $y(x) ; \bar{y}(x ; \alpha)$ follows immediately with the constant $\alpha_{j}, j=0, \overline{1}, 2, \ldots, N-1$ are as yet undetermined. The solution of the fuzzy delay differential equation in form (33) can be determined by the series (37) and (38) with the iterations (45) and (46). 
Define

$$
\begin{aligned}
& {[F(x, y(x), y(x-t)) ; \alpha]} \\
& \quad=F[x, \underline{y}(x ; \alpha), \bar{y}(x ; \alpha), \underline{y}(x-t ; \alpha), \bar{y}(x-t ; \alpha)], \\
& {[G(x, y(x), y(x-t)) ; \alpha]} \\
& \quad=G[x, \underline{y}(x ; \alpha), \bar{y}(x ; \alpha), \underline{y}(x-t ; \alpha), \bar{y}(x-t ; \alpha)] .
\end{aligned}
$$

The exact and approximate solutions at $x_{n}, 0 \leq n \leq N$ are denoted by $\left[Y\left(x_{n}\right)\right]_{\alpha}=\left[Y_{1}\left(\left(x_{n}\right) ; \alpha\right), Y_{2}\left(\left(x_{n}\right) ; \alpha\right)\right]$ and $\left[y\left(x_{n}\right)\right]_{\alpha}=\left[y_{1}\left(\left(x_{n}\right) ; \alpha\right), y_{2}\left(\left(x_{n}\right) ; \alpha\right)\right]$, respectively. By (33) and (45), (46) we have

$$
\begin{aligned}
& Y_{1}\left(x_{n+1} ; \alpha\right) \approx \sum_{j=0}^{N-1} \frac{\alpha_{j}}{j !} x^{j}+L^{-1} F\left(\sum_{n=0}^{\infty} A_{n}\right) ; \\
& Y_{2}\left(x_{n+1} ; \alpha\right) \approx \sum_{j=0}^{N-1} \frac{\alpha_{j}}{j !} x^{j}+L^{-1} G\left(\sum_{n=0}^{\infty} B_{n}\right) .
\end{aligned}
$$

We define

$$
\begin{aligned}
& y_{1}\left(x_{n+1} ; \alpha\right)=\sum_{j=0}^{N-1} \frac{\alpha_{j}}{j !} x^{j}+L^{-1} F\left(\sum_{n=0}^{\infty} A_{n}\right) ; \\
& y_{2}\left(x_{n+1} ; \alpha\right)=\sum_{j=0}^{N-1} \frac{\alpha_{j}}{j !} x^{j}+L^{-1} G\left(\sum_{n=0}^{\infty} B_{n}\right) .
\end{aligned}
$$

The following lemmas will be applied to show convergence of these approximates. That is,

$$
\begin{aligned}
& \lim _{n \rightarrow 0} y_{1}(x ; \alpha)=Y_{1}(x ; r), \\
& \lim _{n \rightarrow 0} y_{2}(x ; \alpha)=Y_{2}(x ; r) .
\end{aligned}
$$

Lemma 11 (see [5]). Let the sequence of numbers $\left\{W_{n}\right\}_{n=0}^{N}$ satisfy

$$
\left|W_{n+1}\right| \leq A\left|W_{n}\right|+B, \quad 0 \leq n \leq N-1,
$$

for some given positive constants $A$ and $B$. Then

$$
\left|W_{n}\right| \leq A^{n}\left|W_{0}\right|+B \frac{A_{n}-1}{A-1}, \quad 0 \leq n \leq N .
$$

Lemma 12 (see [5]). Let the sequence of numbers $\left\{W_{n}\right\}_{n=0}^{N}$, $\left\{V_{n}\right\}_{n=0}^{N}$ satisfy

$$
\begin{gathered}
\left|W_{n+1}\right| \leq A\left|W_{n}\right|+A \max \left\{\left|W_{n}\right|,\left|V_{n}\right|\right\}+B, \\
\left|V_{n+1}\right| \leq A\left|V_{n}\right|+A \max \left\{\left|W_{n}\right|,\left|V_{n}\right|\right\}+B,
\end{gathered}
$$

for some given positive constants $A$ and $B$, and denote

$$
U_{n}=\left|W_{n}\right|+\left|V_{n}\right|, \quad 0 \leq n \leq N .
$$

Then

$$
U_{n}=\bar{A}^{n} U_{0}+\bar{B} \frac{\bar{A}^{n}-1}{\bar{A}-1}, \quad 0 \leq n \leq N,
$$

where $\bar{A}=1+2 A$ and $\bar{B}=2 A$.
Proof. Let $F(t, u, v)$ and $G(t, u, v)$ be obtained by substituting $[y(x)]_{\alpha}=[u, v](46)$

$$
\begin{aligned}
\underline{y}_{0}(x ; \alpha) & =\sum_{j=0}^{N-1} \frac{\alpha_{j}}{j !} x^{j}, \\
\underline{y}_{n+1}(x ; \alpha) & =L^{-1}\left(A_{n}\right), \quad n \geq 0 . \\
\bar{y}_{0}(x ; \alpha) & =\sum_{j=0}^{N-1} \frac{\alpha_{j}}{j !} x^{j}, \\
\bar{y}_{n+1}(x ; \alpha) & =L^{-1}\left(B_{n}\right), \quad n \geq 0 .
\end{aligned}
$$

The domain where $F$ and $G$ are defined is therefore

$$
\begin{aligned}
K & =\{(x, u, v) \mid 0 \leq x \leq t,-\infty<u<\infty,-\infty<v \\
& <\infty\} .
\end{aligned}
$$

Theorem 13 (see [33]). $F(t, u, v)$ and $G(t, u, v)$ belong to the Adomian polynomials $A_{K}, B_{K}$ and let the partial derivatives of $F, G$ be bounded over $K$. Then, for arbitrary fixed $\alpha, 0 \leq$ $\alpha \leq 1$, the approximates of (46) converge to the exact solutions $Y_{1}(x ; \alpha)$ and $Y_{2}(x ; \alpha)$ uniformly in $x$.

Proof. In order to verify that $A_{k}, B_{K}$ (12) depends only on $u_{0}, u_{1}, \ldots, u_{k}$, we can express $A_{k}, B_{K}$ as a function of the coefficients of the two series that are composed using a classical theorem of power series composition [34]. We can note that the expression obtained is only used to prove this dependence. The practical formula will be exposed in Section 5.1. We have just proved that $\sum A_{k}, \sum B_{K}$ is a decomposition series. Now if $\sum u_{n}$ is a convergent series, then $\sum A_{k}\left(u_{0} \cdots u_{k}\right), \sum B_{k}\left(u_{0} \cdots u_{k}\right)$ converges and then its sum is $F \circ u^{+}(1)=F(u), G \circ u^{+}(1)=G(u)$; that is to say, that the decomposition series $\sum A_{k}, \sum B_{K}$ weakly converges and that its sum is $F$ and $G$. Now, if $\sum u_{n}$ and $\sum v_{n}$ are two series having the same sum $U$, and if their Adomian's polynomials are, respectively, $A_{k}^{u}, A_{k}^{v}$ and $B_{k}^{u}, B_{k}^{v}$ then we can write

$$
\begin{aligned}
\sum_{k=0}^{\infty} A_{k}^{u} & =F \circ u^{+}(\lambda=1)=F(U)=F \circ v^{+}(\lambda=1) \\
& =\sum_{k=0}^{\infty} A_{k}^{v}, \\
\sum_{k=0}^{\infty} B_{k}^{u} & =G \circ u^{+}(\lambda=1)=G(U)=G \circ v^{+}(\lambda=1) \\
& =\sum_{k=0}^{\infty} B_{k}^{v} .
\end{aligned}
$$

So, the sum of the Adomian decomposition series depends only on the sum of the considered series: the convergence is strong. 
5.2. Numerical Example. Here, we solve the linear delay differential equations with fuzzy initial conditions to find approximate solutions and tabulated with graphical representation, to the analysis.

Example 1. Consider the linear fuzzy delay differential equation

$$
y^{\prime}(x)=\frac{1}{2} e^{x / 2} y\left(\frac{x}{2}\right)+\frac{1}{2} y(x)
$$

subject to initial conditions $y(0)=1$, then by Definition 3 , we have $y(0)=(\alpha ; 2-\alpha)$.

The exact solution of (59) is

$$
\begin{aligned}
& \underline{y}(x ; \alpha)=\alpha e^{x}, \\
& \bar{y}(x ; \alpha)=(2-\alpha) e^{x} .
\end{aligned}
$$

To approximate (59) by the Adomian decomposition method, it can be written in an operator form

$$
\begin{aligned}
& L \underline{y}(x)=\frac{1}{2} e^{x / 2} \underline{y}\left(\frac{x}{2}\right)+\frac{1}{2} \underline{y}(x), \\
& L \bar{y}(x)=\frac{1}{2} e^{\frac{x}{2}} \bar{y}\left(\frac{x}{2}\right)+\frac{1}{2} \bar{y}(x),
\end{aligned}
$$

with the initial conditions

$$
\begin{aligned}
& \underline{y}(0)=\alpha, \\
& \bar{y}(0)=2-\alpha,
\end{aligned}
$$

where $L=d / d x$ is the differential operator. By operating the two sides of above equation with the inverse operator (namely, $L^{-1}(*)=\int(*) d x$ ) and using the given initial conditions, we have

$$
\begin{aligned}
& \underline{y}(x ; \alpha)=\alpha+\int\left(\frac{1}{2} e^{x / 2} \underline{y}\left(\frac{x}{2}\right)+\frac{1}{2} \underline{y}(x)\right) d x, \\
& \bar{y}(x ; \alpha)=(2-\alpha)+\int\left(\frac{1}{2} e^{x / 2} \bar{y}\left(\frac{x}{2}\right)+\frac{1}{2} \bar{y}(x)\right) d x .
\end{aligned}
$$

Then we obtain the recursive relationship

$$
\begin{aligned}
\underline{y}_{0}(x ; \alpha) & =\alpha, \\
\underline{y}_{n+1}(x ; \alpha) & =\int\left(\frac{1}{2} e^{x / 2} \underline{y}_{n}\left(\frac{x}{2}\right)+\frac{1}{2} \underline{y}_{n}(x)\right) d x, \\
\bar{y}_{0}(x ; \alpha) & =2-\alpha, \\
\bar{y}_{n+1}(x ; \alpha) & =\int\left(\frac{1}{2} e^{\frac{x}{2}} \bar{y}_{n}\left(\frac{x}{2}\right)+\frac{1}{2} \bar{y}_{n}(x)\right) d x .
\end{aligned}
$$

TABLE 1: Exact solution.

\begin{tabular}{lcc}
\hline$\alpha$ & $\underline{y}(x)$ & $\bar{y}(x)$ \\
\hline 0 & 0 & 5.43656364 \\
0.2 & 0.54365637 & 4.89290729 \\
0.4 & 1.08731273 & 4.34925093 \\
0.6 & 1.63096910 & 3.80559456 \\
0.8 & 2.17462546 & 3.26193819 \\
1.0 & 2.71828183 & 2.71828182 \\
\hline
\end{tabular}

Hence we have

$$
\begin{aligned}
& \underline{y_{0}}=\alpha \\
& \underline{y_{1}}=\frac{1}{2} \alpha\left(-2+2 e^{x / 2}+x\right) \\
& \underline{y_{2}}=\frac{1}{24} \alpha\left(-4+16 e^{3 x / 4}(-2+x)-12 x+3 x^{2}\right)
\end{aligned}
$$

$$
\begin{aligned}
\overline{y_{0}} & =(2-\alpha) \\
\overline{y_{1}} & =-\frac{1}{2}(2-\alpha)\left(-2+2 e^{x / 2}+x\right) \\
\overline{y_{2}} & =-\frac{1}{24}(2-\alpha) \\
& \cdot\left(-4+16 e^{3 x / 4}(-2+x)-12 x+3 x^{2}\right)
\end{aligned}
$$

Consequently, the series solution is found as

$$
\begin{aligned}
& \underline{y}(x)=\alpha+\frac{1}{2} \alpha\left(-2+2 e^{x / 2}+x\right) \\
& +\frac{1}{24} \alpha\left(-4+16 e^{3 x / 4}(-2+x)-12 x+3 x^{2}\right)+\cdots, \\
& \bar{y}(x)=(2-\alpha)-\frac{1}{2}(2-\alpha)\left(-2+2 e^{x / 2}+x\right) \\
& -\frac{1}{24}(2-\alpha)\left(-4+16 e^{3 x / 4}(-2+x)-12 x+3 x^{2}\right) \\
& +\cdots .
\end{aligned}
$$

The exact solution of (59) subject to the initial condition is (60); the comparison of the exact solution Table 1 and the approximate solution Table 2 is given in Table 3 (Error estimation). The approximate solution is calculated at 25 iterations. Figure 2 shows the graphical representation of the approximate solutions which is very quite close to the exact solution.

\section{Conclusion}

In this paper, we have introduced an algorithm for finding approximate solutions of delay differential equations with 
TABLE 2: Approximate solution.

\begin{tabular}{lcc}
\hline$\alpha$ & $\underline{y}(x)$ & $\bar{y}(x)$ \\
\hline 0 & 0 & 5.43656364 \\
0.2 & 0.54365584 & 4.89290729 \\
0.4 & 1.08731168 & 4.34925093 \\
0.6 & 1.63096756 & 3.80559456 \\
0.8 & 2.17462335 & 3.26193819 \\
1.0 & 2.71827919 & 2.71828182 \\
\hline
\end{tabular}

TABLE 3: Error estimation.

\begin{tabular}{lcc}
\hline$\alpha$ & $\underline{y}(x)$ & $\bar{y}(x)$ \\
\hline 0 & 0 & $1.69181 \times 10^{-8}$ \\
0.2 & $5.2727810 \times 10^{-7}$ & $1.2263 \times 10^{-9}$ \\
0.4 & $1.0545563 \times 10^{-6}$ & $5.5345 \times 10^{-9}$ \\
0.6 & $1.5818344 \times 10^{-6}$ & $9.8442 \times 10^{-9}$ \\
0.8 & $2.1091126 \times 10^{-6}$ & $4.1509 \times 10^{-9}$ \\
1 & $2.6363907 \times 10^{-6}$ & $8.459 \times 10^{-9}$ \\
\hline
\end{tabular}

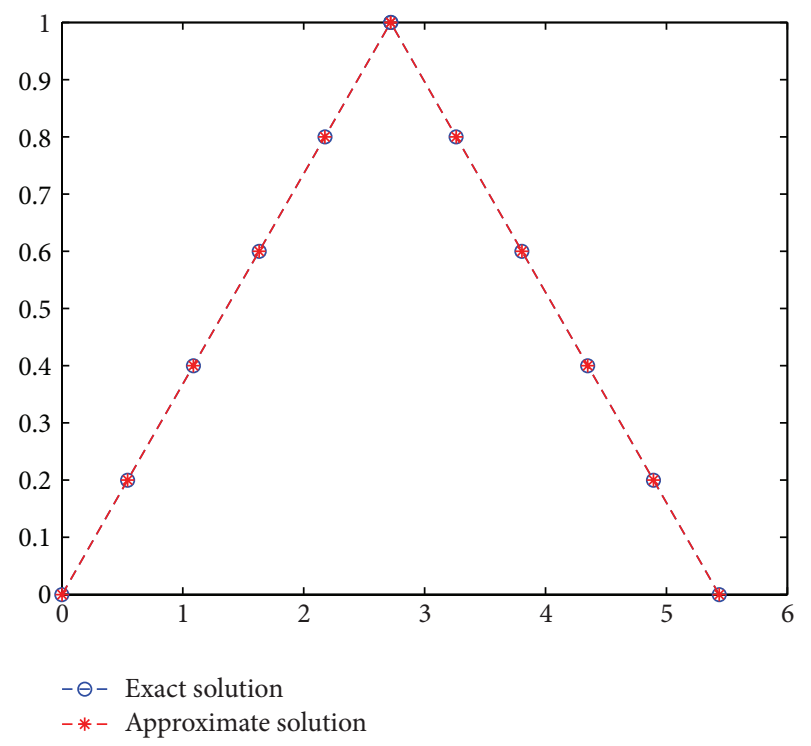

FIGURE 2: Graph at $x=1$.

Adomian decomposition method. For illustration purposes, an example is selected to show the computational accuracy. It may be concluded that the Adomian decomposition method is very powerful and efficient in finding approximate solutions for wide classes of problems. The solutions obtained by the present method are uniformly convergent. As shown in Tables 1-3, results of numerical example show that the present method is an accurate and reliable analytical method for these problems. The present study has confirmed that the Adomian decomposition method offers significant advantages in terms of its straightforward applicability, its computational effectiveness, and its accuracy to solve the strongly linear equations. The Mathematica package 5.2 is used to solve the fuzzy delay differential equations.

\section{Conflict of Interests}

The authors declare that they have no conflict of interests.

\section{Authors' Contribution}

The authors contributed equally and significantly in writing this paper. All authors read and approved the final paper.

\section{Acknowledgments}

The authors would like to thank the Editor and anonymous referees for their helpful suggestions and comments.

\section{References}

[1] O. Kaleva, "The cauchy problem for fuzzy differential equations," Fuzzy Sets and Systems, vol. 35, no. 3, pp. 389-396, 1990.

[2] O. Kaleva, "Fuzzy differential equations," Fuzzy Sets and Systems, vol. 24, no. 3, pp. 301-317, 1987.

[3] L. A. Zadeh, "Fuzzy sets," Information and Control, vol. 8, no. 3, pp. 338-353, 1965.

[4] S. L. Chang and L. A. Zadeh, "On fuzzy mapping and control," IEEE Transactions on Systems, Man and Cybernetics, vol. 2, no. 1, pp. 30-34, 1972.

[5] M. Ma, M. Friedman, and A. Kandel, "Numerical solutions of fuzzy differential equations," Fuzzy Sets and Systems, vol. 105, no. 1, pp. 133-138, 1999.

[6] R. Gethsi Sharmila and E. C. Henry Amirtharaj, "Numerical solution of $N^{\text {th }}$ order fuzzy initial value problems by fourth order Runge-Kutta method based on centroidal mean," IOSR Journal of Mathematics, vol. 3, no. 3, pp. 47-63, 2013.

[7] K. Kanagarajan and M. Sambath, "Numerical solution of fuzzy differential equations by third order Runge-Kutta method," International Journal of Applied Mathematics and Computation, vol. 2, no. 4, pp. 1-8, 2010.

[8] O. Abu-Arqub, A. El-Ajou, S. Momani, and N. Shawagfeh, "Analytical solutions of fuzzy initial value problems by HAM," Applied Mathematics and Information Sciences, vol. 7, no. 5, pp. 1903-1919, 2013.

[9] S. Abbasbandy and T. Allahviranloo, "Numerical solution of Fuzzy delay differential equations by Taylor method," Journal of Computational Methods in Applied Mathematics, vol. 2, pp. 113-124, 2002.

[10] S. Seikkala, "On the fuzzy initial value problem," Fuzzy Sets and Systems, vol. 24, no. 3, pp. 319-330, 1987.

[11] H. Jafari, M. Saeidy, and D. Baleanu, "The variational iteration method for solving n-th order fuzzy differential equations," Central European Journal of Physics, vol. 10, no. 1, pp. 76-85, 2012.

[12] T. Allahviranloo, N. Ahmady, and E. Ahmady, "Numerical solution of fuzzy differential equations by predictor-corrector method," Information Sciences, vol. 177, no. 7, pp. 1633-1647, 2007.

[13] A. D. Myškis, Linear Differential Equations with Retarded Argument, Izdat, Nauka, Moscow, Russia, 2nd edition, 1972, (revised and enlarged version of the 1951 original). MR 14-52, MR 17-497 and MR 50 \# 5135.

[14] R. Bellman and K. L. Cooke, Differential-Difference Equations, Academic Press, New York, NY, USA, 1963. 
[15] R. D. Driver, Ordinary and Delay Differential Equations, Springer, New York, NY, USA, 1977.

[16] A. Bellen and M. Zennaro, Numerical Methods for Delay Differential Equations, Clarendon Press, Oxford, UK, 2003.

[17] A. Khastan, J. J. Nieto, and R. Rodríguez-López, "Fuzzy delay differential equations under generalized differentiability," Information Sciences, vol. 275, pp. 145-167, 2014.

[18] K. Barzinji, N. Maan, and N. Aris, "Linear fuzzy delay differential systems: analysis a stability of steady state," Matematika, vol. 30, pp. 1-7, 2014.

[19] M. A.-K. Ibrahim, A. El-Safty, and S. M. Abo-Hasha, "2h-Step spline method for the solution of delay differential equations," Computers \& Mathematics with Applications, vol. 29, no. 8, pp. 1-6, 1995.

[20] D. J. Evans and K. R. Raslan, "The Adomian decomposition method for solving delay differential equation," International Journal of Computer Mathematics, vol. 82, no. 1, pp. 49-54, 2005.

[21] F. Shakeri and M. Dehghan, "Solution of delay differential equations via a homotopy perturbation method," Mathematical and Computer Modelling, vol. 48, no. 3-4, pp. 486-498, 2008.

[22] F. Mirzaee and L. Latifi, "Numerical solution of delay differential equations by differential transform method," Journal of Sciences (Islamic Azad University), vol. 20, pp. 83-88, 2011.

[23] M. Sadeghi Hafshejani, S. Karimi Vanani, and J. Sedighi Hafshejani, "Numerical solution of delay differential equations using Legendre wavelet method," World Applied Sciences Journal, vol. 13, pp. 27-33, 2011.

[24] M. M. Khader, "Numerical and theoretical treatment for solving linear and nonlinear delay differential equations using variational iteration method," Arab Journal of Mathematical Sciences, vol. 19, no. 2, pp. 243-256, 2013.

[25] L. Guerrini, "Delay differential AK model with non-positive population growth rate," Applied Mathematical Sciences, vol. 6, no. 69-72, pp. 3575-3577, 2012.

[26] C. Bianca, M. Ferrara, and L. Guerrini, "The time delays effects on the qualitative behavior of an economic growth model," Abstract and Applied Analysis, vol. 2013, Article ID 901014, 10 pages, 2013.

[27] A. Akgül and A. Kiliçman, "Solving delay differential equations by an accurate method with interpolation," Abstract and Applied Analysis, vol. 2015, Article ID 676939, 7 pages, 2015.

[28] N. H. Abdul Aziz, Z. Abdul Majid, and F. Ismail, "Solving delay differential equations of small and vanishing lag using multistep block method," Journal of Applied Mathematics, vol. 2014, Article ID 348912, 10 pages, 2014.

[29] G. Adomian, Solving Frontier Problems of Physics: The Decomposition Method, Kluwer Academic Publishers, 1994.

[30] D. Dubois and H. Prade, "Operations on fuzzy number," International Journal of Systems Science, vol. 9, no. 6, pp. 613626, 1978

[31] G. Adomian, "A review of the decomposition method in applied mathematics," Journal of Mathematical Analysis and Applications, vol. 135, no. 2, pp. 501-544, 1988.

[32] G. Adomian and R. Rach, "Generalization of adomian polynomials to functions of several variables," Computers and Mathematics with Applications, vol. 24, no. 5-6, pp. 11-24, 1992.

[33] L. Gabet, "The theoretical foundation of the Adomian method," Computers and Mathematics with Applications, vol. 27, no. 12, pp. 41-52, 1994.
[34] Y. Cherruault, G. Saccomandi, and B. Some, "New results for convergence of Adomian's method applied to integral equations," Mathematical and Computer Modelling, vol. 16, no. 2, pp. 83-93, 1992. 


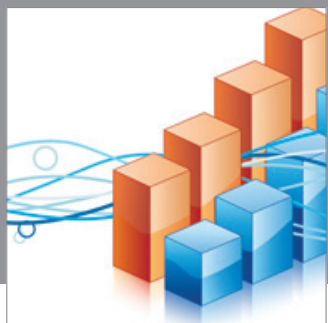

Advances in

Operations Research

mansans

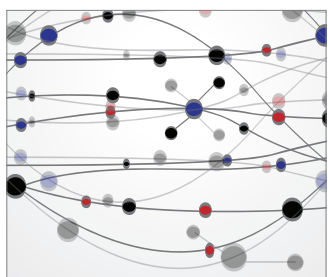

The Scientific World Journal
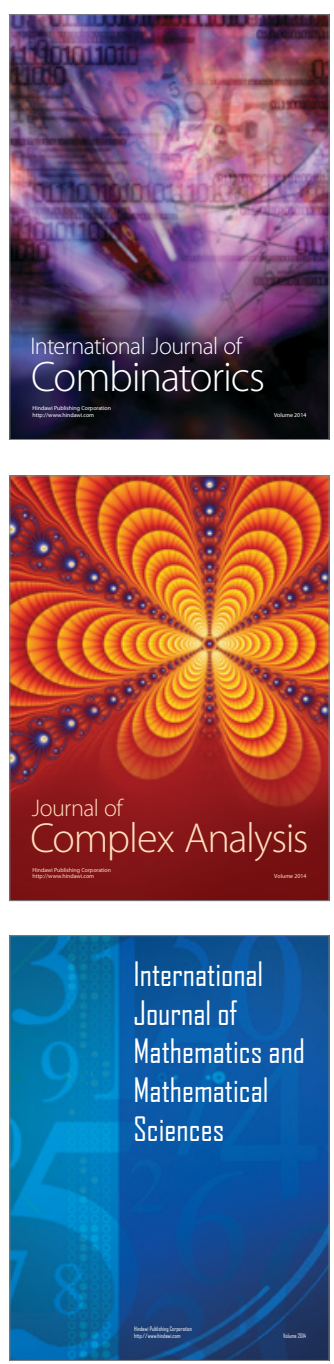
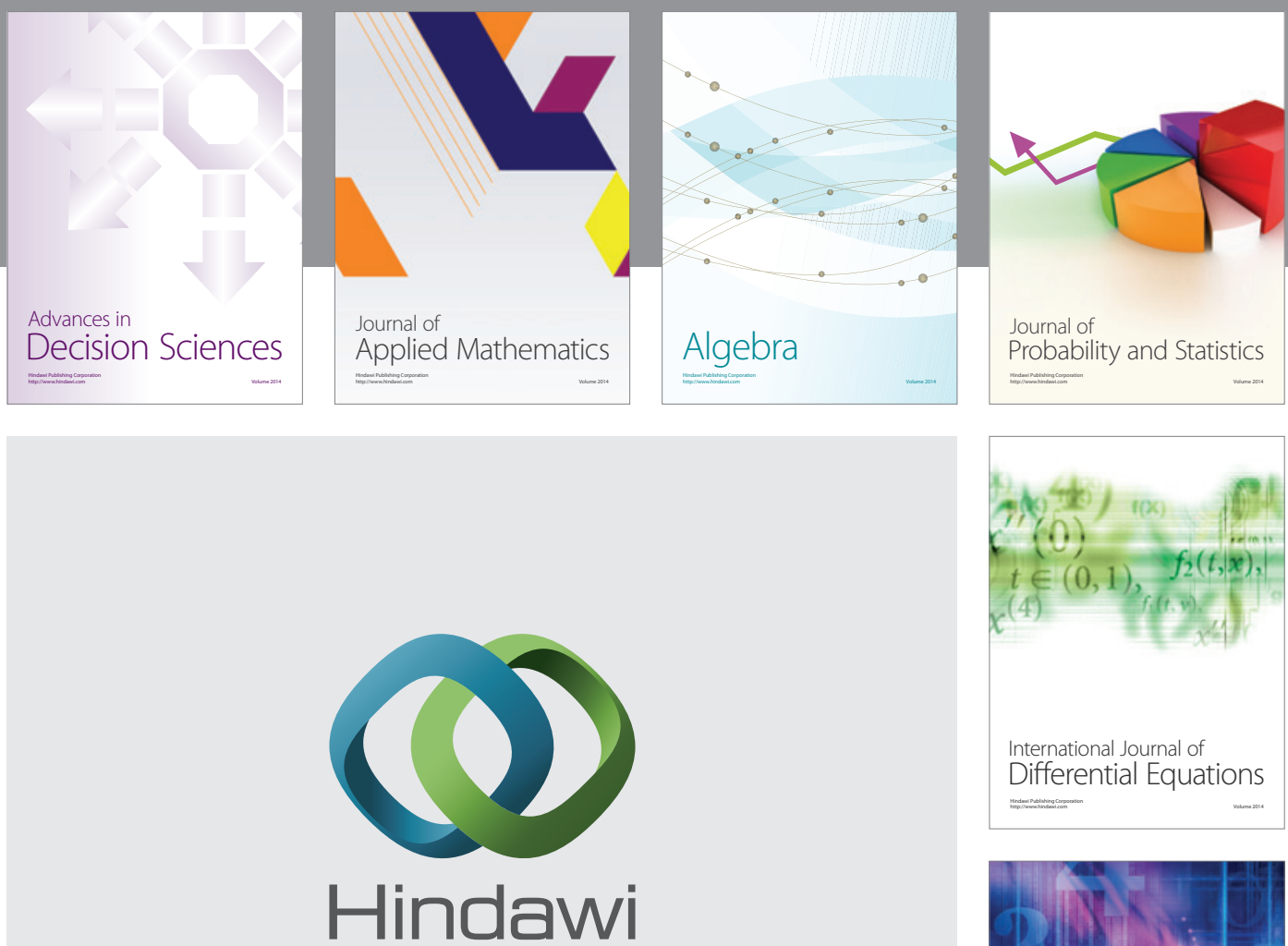

Submit your manuscripts at http://www.hindawi.com
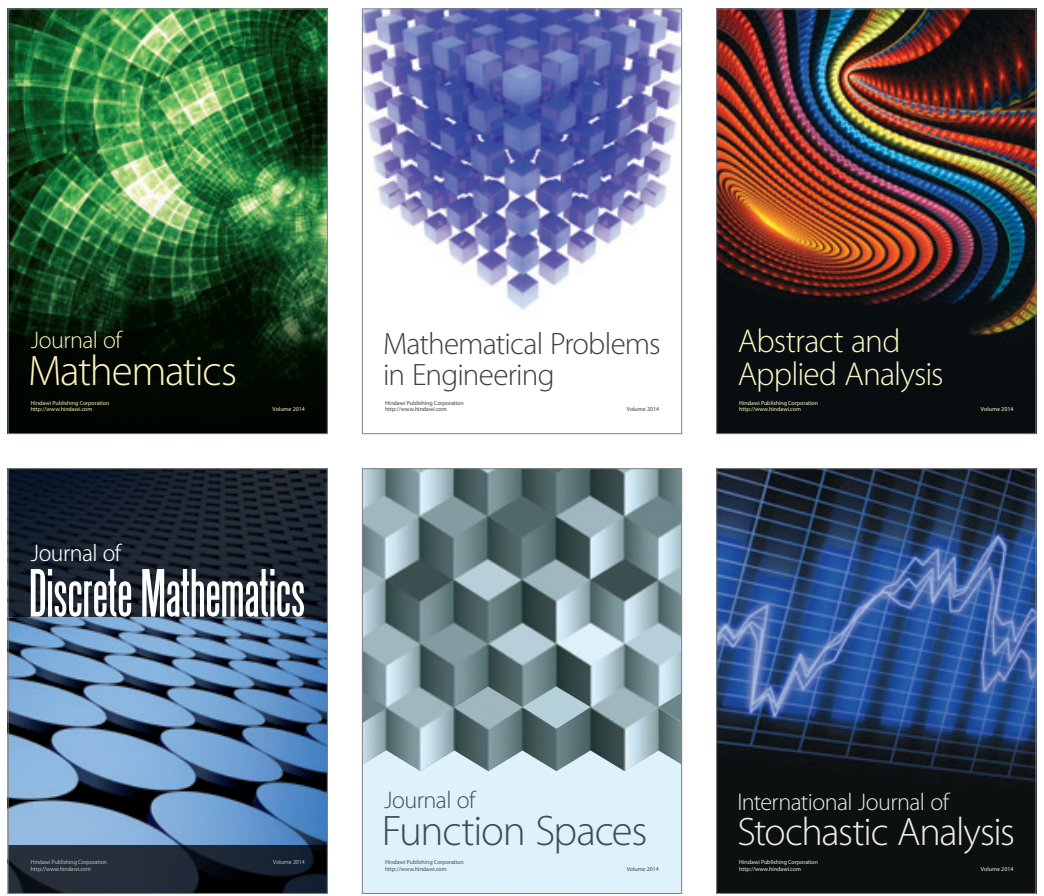

Journal of

Function Spaces

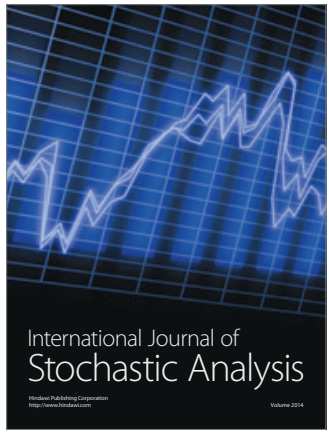

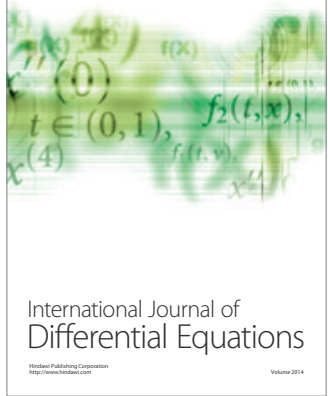
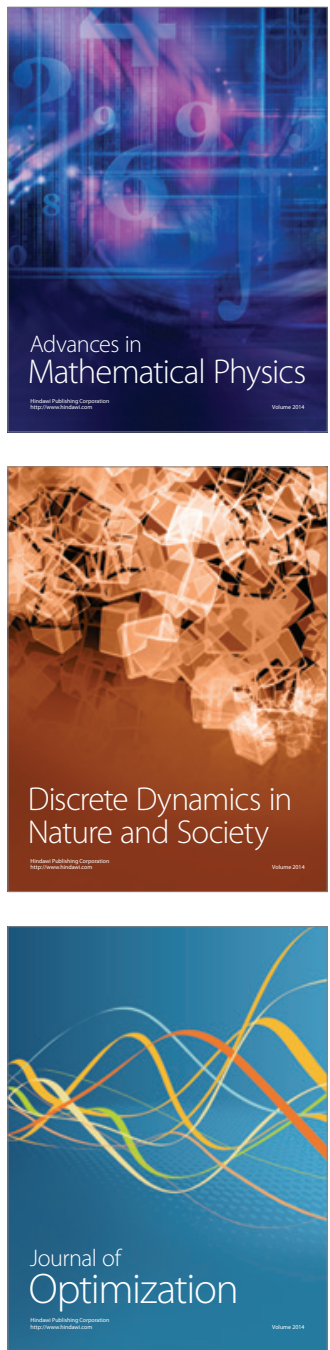